\title{
Monitoring of platelet function parameters and microRNA expression levels in patients with prostate cancer treated with volumetric modulated arc radiotherapy
}

\author{
NURTEN BAHTIYAR ${ }^{1}$, ILHAN ONARAN ${ }^{2}$, BIRSEN AYDEMIR $^{3}$, ONUR BAYKARA $^{2}$, \\ SELMIN TOPLAN $^{1}$, FULYA YAMAN AGAOGLU ${ }^{4}$ and MEHMET CAN AKYOLCU ${ }^{1}$ \\ Departments of ${ }^{1}$ Biophysics and ${ }^{2}$ Medical Biology, Cerrahpasa Faculty of Medicine, Istanbul University, \\ Istanbul 34098; ${ }^{3}$ Department of Biophysics, Faculty of Medicine, Sakarya University, Sakarya 54050; \\ ${ }^{4}$ Department of Radiation Oncology, Institute of Oncology, Istanbul University, Istanbul 34098, Turkey
}

Received July 12, 2017; Accepted November 16, 2017

DOI: $10.3892 / \mathrm{ol} .2018 .9167$

\begin{abstract}
Radiotherapy (RT) may result in platelet activation and thrombosis development. To the best of our knowledge, the potential effect of volumetric-modulated arc therapy (VMAT), a novel radiotherapy technique, on platelet function and microRNA (miRNA/miR) expression has not been previously investigated. The present study aimed to determine the effect of VMAT on the alterations in platelet function parameters and miRNA expression levels. A total of 25 patients with prostate cancer and 25 healthy subjects were included in the present study. Blood samples were collected from the patient group on the day prior to RT (pre-RT), the day RT was completed (post-RT day 0), and 40 days following the end of therapy (post-RT day 40). Platelet count, mean platelet volume (MPV) value, platelet aggregation, plasma P-selectin, thrombospondin-1, platelet factor 4, plasma miR-223 and miR-126 expression levels were measured. A significant decrease in platelet count in the post-RT day 0 group was measured in comparison with the pre-RT and the post-RT day 40 groups. Pre-RT MPV values were higher than those of the post-RT day 0 and the post-RT day 40 groups. No significant differences were observed in the levels of platelet activation markers or miR-223 and miR-126 expression levels between the RT groups. Although RT may result in a reduction in platelet and MPV counts, the results of the present study indicate that platelet activation markers are not affected by VMAT. Therefore, it is possible that no platelet activation occurs during VMAT, owing to the conformal dose distributions, improved
\end{abstract}

Correspondence to: Dr Nurten Bahtiyar, Department of Biophysics, Cerrahpasa Faculty of Medicine, Istanbul University, 53 Kocamustafa Pasa Street, Fatih, Istanbul 34098, Turkey

E-mail: nurtenbahtiyar@hotmail.com

Key words: radiotherapy, volumetric modulated arc therapy, prostate cancer, platelet function, microRNA expression target volume coverage and the sparing of normal tissues from undesired radiation.

\section{Introduction}

Prostate cancer was a common global malignancy among men in $2012(1,2)$. Ionizing radiation is used to treat various types of cancer (3), including localized prostate cancer (4). External beam radiotherapy (RT) has been demonstrated to be a curative method for localized prostate cancer (5) by the European Association of Urology guidelines (6).

In addition to killing cancerous cells, ionizing radiation damages healthy cells. The hematopoietic system is an important system in radiosensitive patients who are affected by the acute-phase effects of radiation (7). Ionizing radiation results in vascular damage through exertion of a cytotoxic effect on the vascular endothelium $(8,9)$. Furthermore, homeostatic responses are activated due to oxidative damage that develops in the endothelium, including the activation of platelets in irradiated tissues, which results in platelet aggregation (9). The probability of thrombosis differs based on the type of cancer, with this probability varying between 6.9 and $11.4 \%$ in prostate cancer (10). Platelets are known to serve notable functions in the formation of thrombosis $(11,12)$.

Since the mid-90s, developments in techniques used in RT have allowed researchers to increase control over the tumor while decreasing the side effects that result from the treatment. These developments have additionally benefitted radiation therapy used for prostate cancer (5). A previous study performed with three-dimensional conformal RT (3D-CRT) in prostate cancer revealed that toxicity may be decreased if the target and the RT volume are compatible (6). Intensity-modulated RT (IMRT), a technique used in 3D-CRT, is the most common method used to treat prostate cancer. This method provides a better distribution of conformal doses, thus increasing the targeted dose distribution and decreasing the toxicity effect on normal tissues (5). A recently developed technique named volumetric-modulated arc therapy (VMAT) delivers the radiation dose more efficiently with a dynamic modulated arc $(5,13)$; however, there are a limited number of 
studies focusing on the effect on platelet functions in acute and chronic phases of radiation therapy applied using the VMAT technique. Studies performed on the effects of RT on platelet functions usually focus on specific markers, including platelet count and mean platelet volume (MPV) (7,14-16). In addition to platelet aggregation (17), the levels of P-selectin $(18,19)$, thrombospondin-1 (20) and platelet factor 4 (PF 4) (21) are less commonly used parameters as markers of platelet function.

Previous molecular studies have revealed an association between serum/plasma levels and platelet levels and a number of microRNAs (miRNA/miRs), which direct gene expression by targeting mRNA at the post-transcriptional level $(22,23)$. As miRNA expression levels may be altered in various types of disease, these tissue-specific molecules may be used as disease markers by measuring their levels in circulation (24). It has been suggested that a number of miRNAs may additionally be used as platelet activation markers (25-27).

A number of miRNAs are associated with platelet function, miR-223 and miR-126, which is expressed in platelets, are among the most commonly studied molecules. One previous study revealed that miR-223 and miR-126 serve a critical function in regulating the expression of vascular cell adhesion protein 1 and $\mathrm{P} 2 \mathrm{Y} 12$ receptors in platelets (25). It has been reported that the altered plasma levels of these two miRNAs in cardiovascular diseases is associated with platelet function $(24,26,27)$. This change may be plausible in post-treatment serum/plasma levels as a result of miRNAs released from platelets due to the toxic effect of RT. However, to the best of our knowledge, no studies have investigated this type of change caused by treatment with RT, particularly VMAT, on patients with prostate or other types of cancer. In the light of the aforementioned information, the present study was conducted to analyze the effect of VMAT on platelet function in patients with prostate cancer using previously determined markers, and to evaluate the association of plasma levels of miR-223 and miR-126 with treatment.

\section{Materials and methods}

Case selection. A total of 25 male patients (mean age 66.57 , range 55-76 years) diagnosed with histologically confirmed adenocarcinoma of the prostate and treated with RT at the Istanbul University Oncology Institute, Department of Radiation Oncology (Istanbul, Turkey) between June 2013 and February 2014 were included in the study. A total of 25 healthy male volunteers of similar age (mean age 63.76, range 52-76 years), who did not receive any medication, constituted the control group. Medical history, including hematological disorders that affect platelet count or platelet function, distant metastases or other malignant diseases, diabetes, hypertension, chronic inflammatory diseases, infectious diseases and autoimmune diseases, were designated as exclusion criteria for the patients with prostate cancer. A known history of chronic, inflammatory or malignant diseases and the use of antithrombotic drugs were the exclusion criteria for the healthy control subjects. Patients with prostate cancer received therapy with VMAT (Varian Trilogy Rapid Arc Radiotherapy Device; Varian Medical Systems, Inc., Palo Alto, CA, USA) with a daily dose of 180-200 cGy fractions, 5 days a week for 30-37 days. The doses of the planned target volumes for prostate and seminal vesicles were 66-74 and 50-54 Gy, respectively. The total postoperative dose administered to the prostatic fossa was $66 \mathrm{~Gy}$. Whole pelvis irradiation was not given. All patients gave written informed consent, and the present study was approved by the Istanbul University Cerrahpasa Medical Faculty Ethics Committee (approval number: 28052/2012) and the present study was performed in accordance with The Declaration of Helsinki.

Sample collection. Venous blood was collected into tubes containing $3.8 \%$ sodium citrate and EDTA separately from each person the day prior to RT (pre-RT group), the day RT was completed (post-RT day 0 group) and 40 days following the end of RT (post-RT day 40 group) from patients with prostate cancer. The same volume of blood was collected into the tubes from healthy control subjects.

Platelet count and MPV values. Platelet counts and MPV values were determined using Cell-DYN C1600 (Abbott Pharmacuetical Co., Ltd., Lake Bluff, IL, USA) blood count device using blood collected as aforementioned.

Platelet aggregation. Platelet aggregation was determined according to the method of Angiolillo et al (28) using a light transmittance aggregometer (Chrono-log 500; Chrono Log Corp., Havertown, PA, USA). Venous blood samples were collected in tubes containing 3.8\% sodium citrate. Platelet-rich plasma (PRP) was obtained as a supernatant subsequent to centrifugation of citrated blood at $160 \mathrm{x} \mathrm{g}$ for $10 \mathrm{~min}$ at $22^{\circ} \mathrm{C}$. The remaining blood was centrifuged at 2,500 $\mathrm{x}$ g for $10 \mathrm{~min}$ at $22^{\circ} \mathrm{C}$ to obtain platelet poor plasma (PPP). PPP and PRP were incubated for $3 \mathrm{~min}$ at $37^{\circ} \mathrm{C}$. Following incubation, $1 \mu \mathrm{M}$ of adenosine diphosphate (Chrono Log Corp.) was added into PRP to induce the platelet aggregation. Platelet aggregation curves were recorded for $3 \mathrm{~min}$ (29). Results were expressed as the extent of maximal aggregation (\% of maximal amplitude).

Platelet activation. Venous blood samples containing $3.8 \%$ sodium citrate were centrifuged for $15 \mathrm{~min}$ at $1,000 \mathrm{x} \mathrm{g}$ at $4^{\circ} \mathrm{C}$, and the obtained plasma samples were stored at $-80^{\circ} \mathrm{C}$ until plasma thrombospondin-1, P-selectin and PF 4 were measured using an ELISA. Subsequent to the thawing of each plasma sample at $4^{\circ} \mathrm{C}$, the measurements of plasma thrombospondin-1 (EIAab Science Co. Ltd., Wuhan, Hubei, China; cat. no. E0611 h), P-selectin (Wuhan EIAab Science Co. Ltd.; cat. no. E0115 h) and PF 4 (Wuhan EIAab Science Co. Ltd.; cat. no. E0172 h) were performed using commercial kits according to the manufacturer's protocol.

miRNA detection with reverse transcription-quantitative polymerase chain reaction $(R T-q P C R)$. EDTA-plasma samples stored at $-80^{\circ} \mathrm{C}$ were thawed at $4^{\circ} \mathrm{C}$ prior to use and miRNA was immediately isolated by using the Qiagen miRNeasy Serum/Plasma kit (Qiagen GmbH, Hilden, Germany) following manufacturer's protocol.cDNA synthesis was synthesized using ABM miRNA cDNA Synthesis kit with Poly(A) Polymerase Tailing (ABM Inc., Vancouver, Canada) according to manufacturer's protocol. Samples were stored at $-80^{\circ} \mathrm{C}$ until further use. The expression levels of miRNAs were determined using the $\mathrm{Eco}^{\mathrm{TM}}$ Real-Time 
PCR system (Illumina, Inc., San Diego, CA, USA). The primers used were as follows: Homo sapiens (hsa)-miR-223 forward, 5'-GTCCGCTGTCAGTTTGTCAAATAC-3' and reverse, 5'-GTGCGTGTCGTGGAGTC-3'; hsa-miR-126 forward, 5'-GTCCGCTCGTACCGTGAGTAATA-3' and reverse 5'-GTGCGTGTCGTGGAGTC-3'; U6-2 forward 5'-GCCCCTGCGCAAGGATGAC-3' and reverse, 5'-CCA GTGCAGGGTCCGAGGTA-3'. All miRNA primers were procured from ABM Inc. RT-qPCR was conducted by using the EvaGreen miRNA qPCR MasterMix (Applied Biological Materials, Inc., Richmond, BC, Canada). The reaction conditions were as follows: 1 cycle for $10 \mathrm{~min}$ at $95^{\circ} \mathrm{C}, 40$ cycles for $\left(10 \mathrm{sec}\right.$ at $95^{\circ} \mathrm{C}, 15 \mathrm{sec}$ at $60^{\circ} \mathrm{C}$ and $5 \mathrm{sec}$ at $\left.72^{\circ} \mathrm{C}\right)$. Melting curve analysis was performed with a sensitivity of $0.1^{\circ} \mathrm{C}$ at temperatures between 55 and $90^{\circ} \mathrm{C}$. PCR analyses were performed by calculating the standard curve and the number of duplicates. The Eco study software v5.0 (Illumina, Inc.) was used to calculate quantification cycle $(\mathrm{Cq})$ expression values (30) for all genes. The U6-2 small nuclear RNA was used as an internal control to detect hsa-miR-223/miR-U6-2 and hsa-miR-126/miR-U6-2 ratios in the plasma.

Statistical analysis. Data are presented as mean \pm the standard deviation (SD). Statistical analysis was performed using the Wilcoxon signed-rank and Mann-Whitney U-tests. Correlation analysis using Spearman's rank was used to study the association between markers. $\mathrm{P}<0.05$ was considered to indicate a statistically significant difference. Variation coefficiency $(\% \mathrm{Cv}$ ) was used to calculate the scattering due to individual variations. All calculations were performed using GraphPad Prism version 5.00 for Windows (GraphPad Software, Inc., La Jolla, CA, USA). $\mathrm{Cv}$ values were calculated as: $\% \mathrm{Cv}=(\mathrm{SD}$ of measurement/measurement average) x100.

\section{Results}

Patient data. Demographic data of patient and control groups are presented in Table I. Platelet count, MPV value and platelet activation markers are presented in Table II as the mean \pm SD.

Platelet count and MPV value. The platelet count of the post-RT day 0 group was significantly reduced vs. the pre-RT and the post-RT day 40 groups $(208.90 \pm 37.45$ vs. $228.80 \pm 43.39$ and 232.80 $\pm 44.79 ; \mathrm{P}=0.002$ and $\mathrm{P}=0.001$, respectively). There were no significant differences identified $(\mathrm{P}>0.05)$ between the platelet count of the post-RT day 40 group and the pre-RT group, and all RT groups compared with the control group. Platelet count values are presented as the mean \pm SD in Table II.

The MPV values of the pre-RT group were identified to be higher than the post-RT day 0 and post-RT day 40 groups (8.81 \pm 0.81 vs. $8.34 \pm 0.79$ and $8.19 \pm 0.81 ; \mathrm{P}<0.001$ and $\mathrm{P}<0.001$, respectively). No significant difference in MPV values was identified between the post-RT day 0 and post-RT day 40 groups $(\mathrm{P}>0.05)$. Furthermore, there were no significant differences identified $(\mathrm{P}>0.05)$ in the control group compared with the post-RT day 0 and post-RT day 40 groups. However, a significant difference was identified between the pre-RT and control groups $(\mathrm{P}<0.05)$. MPV values are presented as the mean \pm SD in Table II.
Table I. Demographic data of patient and control groups.

\begin{tabular}{lccc}
\hline Variables & Patients & Control & P-value \\
\hline Age, years & $66.57 \pm 6.65^{\mathrm{a}}$ & $63.76 \pm 8.49^{\mathrm{a}}$ & 0.386 \\
Histology & & & \\
Adenocarcinoma, n (\%) & $25(100)$ & $\mathrm{NA}$ & \\
Tumor stage & & & \\
T2c, n (\%) & $4(16 \%)$ & $\mathrm{NA}$ & \\
T3b, n (\%) & $12(48 \%)$ & $\mathrm{NA}$ & \\
T3c, n (\%) & $9(36 \%)$ & $\mathrm{NA}$ & \\
Total radiation dose, cGy & $6,600-7,400$ & $\mathrm{NA}$ & \\
Fractions, $\mathrm{n}$ & $30-37$ & $\mathrm{NA}$ & \\
Family history & & & \\
Yes, n $(\%)$ & $3(12 \%)$ & $\mathrm{NA}$ & \\
No, n $(\%)$ & $22(88 \%)$ & NA & \\
\hline
\end{tabular}

${ }^{\mathrm{a}}$ Mean \pm standard deviation; NA, not applicable.

Platelet activation markers. Platelet aggregation, plasma P-selectin, plasma thrombospondin-1 and plasma PF 4 were measured as platelet activation markers. There was no significant difference $(\mathrm{P}>0.05)$ between RT and control groups and among RT groups when platelet aggregation, plasma levels of P-selectin, thrombospondin-1 and PF 4 were measured (Table II). However, high values of plasma thrombospondin-1 and PF 4 were observed in the coefficient of variation calculations used to determine the scattering due to personal variations (Cv 51.6 to 63.4 and 41.8 to $54.5 \%$, respectively; data not shown).

miR-223 and miR-126 expression. Plasma expression levels of miR-223 and miR-126 are given as Cq miR-223/Cq miR-U6-2 and $\mathrm{Cq}$ miR-126/Cq miR-U6-2 ratios (Fig. 1A and B, respectively). No significant difference $(\mathrm{P}>0.05)$ was identified between the plasma miR-223 expression levels of the RT-treated and control groups and amongst the RT groups. Similarly, the miR-126 expression levels did not vary significantly $(\mathrm{P}>0.05)$ between any of the groups (Table III). Fig. 2A-D shows the individual changes in Cq miR-223/Cq miR U6-2 and Cq miR-126/Cq miR-U6-2 ratios in RT-treated groups. Ct miR-223/Ct miR U6-2 and Ct miR-126/Ct miR U6-2 ratios in patient and control groups values are presented as the mean \pm SD in Table III.

Correlation between miRNA expression levels and platelet function markers. A positive correlation was identified between $\mathrm{Cq}$ miR-223/Cq miR-U6-2 and Cq miR-126/Cq miR-U6-2 ratios in all RT-treated groups. Correlation between Cq miR-223/Cq miR-U6-2 and Cq miR-126/Cq miR-U6-2 ratios in all RT-treated groups are presented in Fig. 3A-C.

\section{Discussion}

RT kills target cells by inducing DNA damage (31). However, RT may also harm the cells of healthy tissues and mitotically active bone marrow as collateral damage (14). Various studies have reported the existence of occlusions in the vascular system in the chronic phase of irradiation and aggregation of 
Table III. Cq miR-223/Cq miR U6-2 ratios in patient and control groups.

\begin{tabular}{lcc}
\hline $\begin{array}{l}\text { Patients } \\
\text { (n=25 per group) }\end{array}$ & $\begin{array}{l}\text { Cq miR-223/ } \\
\text { Cq miR U6-2 }\end{array}$ & $\begin{array}{c}\text { Cq miR-126/ } \\
\text { Cq miR U6-2 }\end{array}$ \\
\hline Pre-RT & $1.17 \pm 0.40$ & $1.15 \pm 0.44$ \\
Post-RT day 0 & $1.31 \pm 0.41$ & $1.22 \pm 0.38$ \\
Post-RT day 40 & $1.23 \pm 0.38$ & $1.17 \pm 0.36$ \\
Control & $1.19 \pm 0.23$ & $1.21 \pm 0.20$ \\
\hline
\end{tabular}

Values are presented as the mean \pm the standard deviation. RT, radiotherapy; miR, microRNA; Cq, quantification cycle.

platelets in the vascular lumen during the $2 \mathrm{~h}$ following irradiation (32-34). Platelet adhesion to vascular wall structures due to radiation results in radiation-associated thrombosis (32).

VMAT was developed as a novel form of arc therapy in 2007 to decrease the side effects of RT (35). There are a number of benefits of VMAT, including increased target specificity and improved healthy tissue avoidance, in addition to improving coverage of the target area by adjusting the gantry rotation and speed, better dose distribution and significantly reduced RT time (36).

Although previous studies have revealed that targeted dose distributions are better managed by VMAT $(36,37)$, to the best of our knowledge there is no concrete data elucidating the effect of VMAT on platelet markers. Therefore, the present study aimed to measure the platelet parameters and activation markers prior to and following RT to determine the effects of radiation administered with VMAT on the platelet count, MPV value, platelet aggregation, levels of P-selectin, thrombospondin- 1 and PF 4 markers. Furthermore, their association with miR-226 and miR-123 expression levels in patients with prostate cancer was also determined.

The results of the present study indicated the presence of a significant difference in platelet count and MPV value in preand post-RT with VMAT treatment groups of patients with prostate cancer. According to the results, the platelet count was decreased by $8.7 \%$ in the post-RT day 0 group in comparison with the pre-RT group and increased by $10 \%$ in the post-RT day 40 group compared with the post-RT day 0 group. Previous studies investigating conventional RT and IMRT techniques have reported a decrease in platelet count following treatment, thus corroborating the results of the present study $(7,15,38,39)$. Furthermore, it has been demonstrated that sub-lethal radiation doses may result in abnormal hemostasis characteristics and coagulation biomarker values observed up to 21 days post-irradiation (40). Therefore, the post-RT decrease in platelet counts observed in the present study may be due to the suppressive effect of radiation on the hematopoietic system in the acute phase. The increase in the platelet count in the post-RT day 40 group may be associated with the removal of the suppressive effect of RT on bone marrow.

MPV is a marker of platelet function and activation. An increase in platelet volume may result in increased platelet activity. The changes in platelet volume may be used as a diagnostic marker for the prevention and follow-up of the 

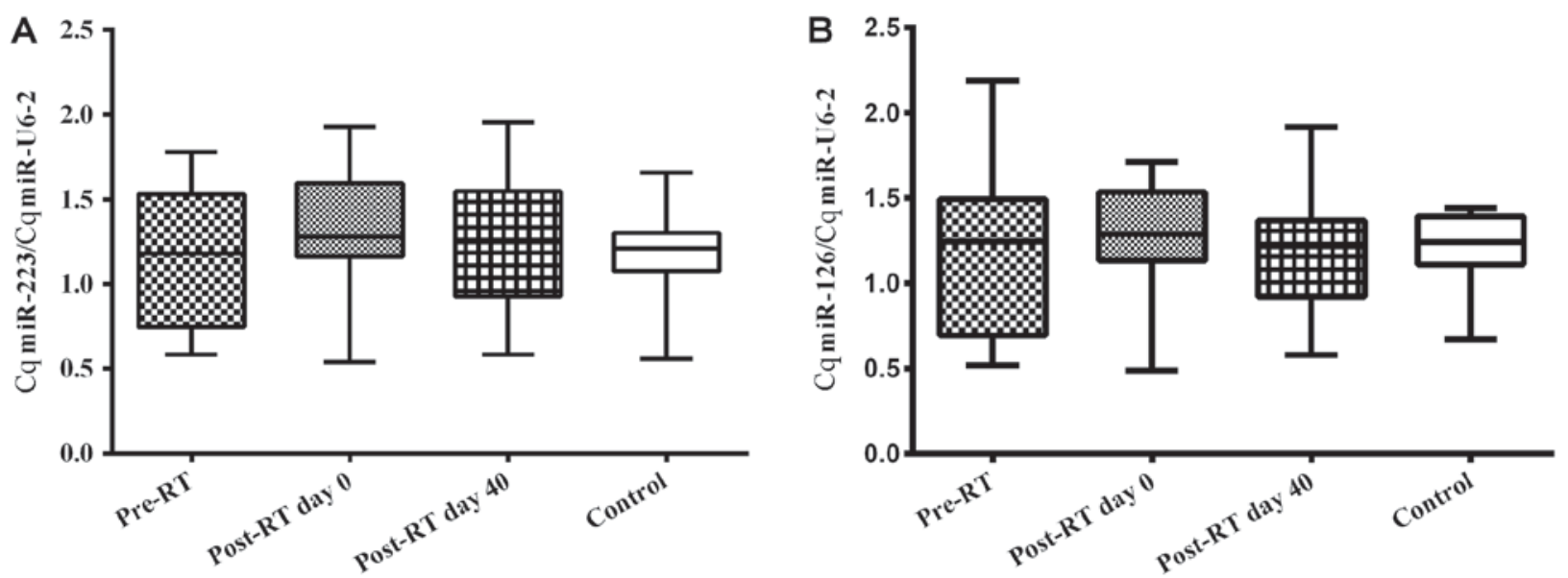

Figure 1. Ratio of miRNAs associated with platelet function in patients and controls. (A) Cq miR-223/Cq miR U6-2 ratios in patients prior to and following RT and control groups. (B) Cq miR-126/Cq miR U6-2 ratios in patients prior to and following RT and control groups. RT, radiotherapy; miR/miRNA, microRNA; $\mathrm{Cq}$, quantification cycle.
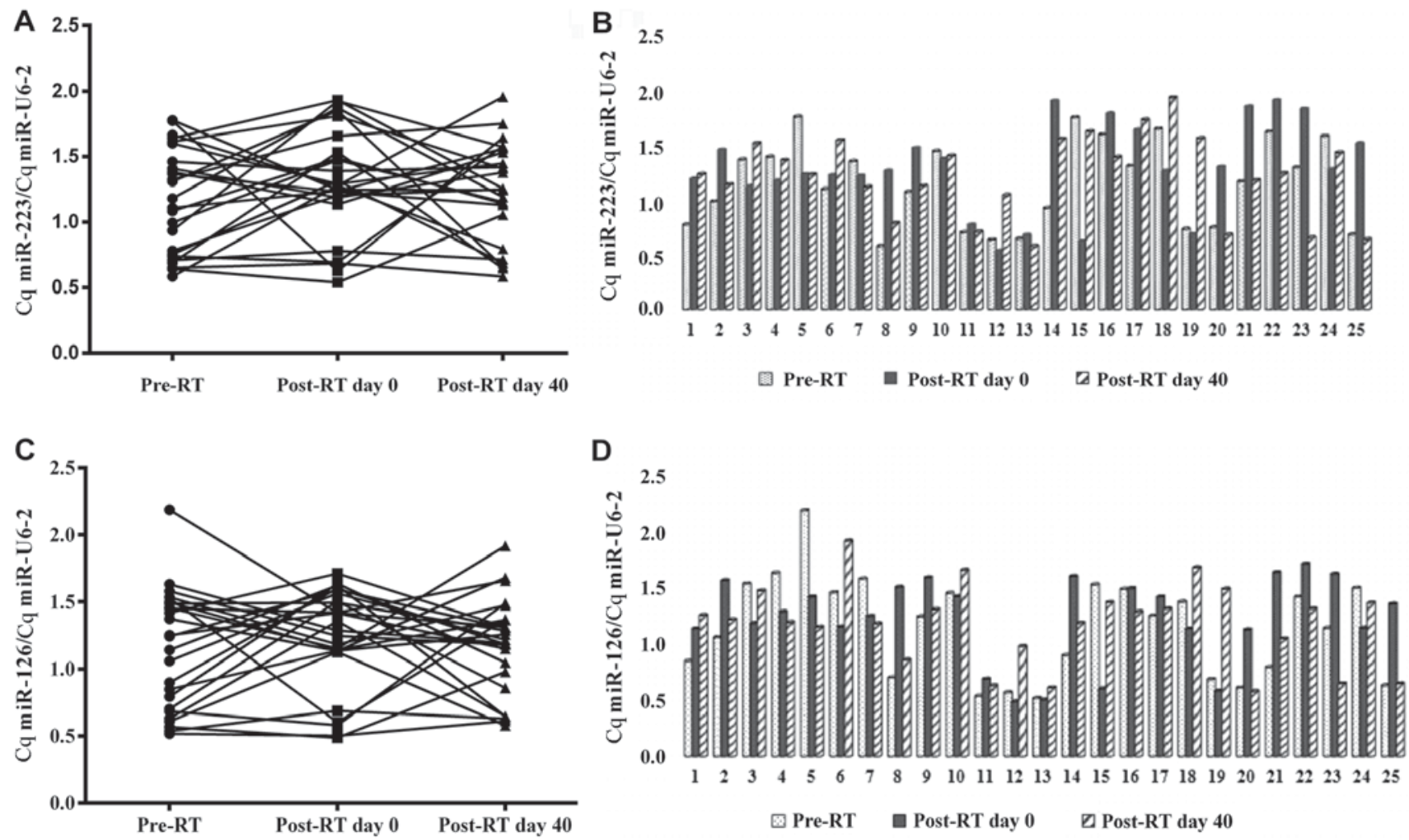

Figure 2. Individual changes to the Cq miR-223/Cq miR U6-2 and Cq miR-126/Cq miR-U6-2 ratios in RT-treated groups. (A and B) Individual changes to Cq miR-223/Cq miR U6-2 ratios in RT-treated groups. (C and D) Individual changes to Cq miR-126/Cq miR U6-2 ratios in RT-treated groups. RT, radiotherapy; miR, microRNA; Cq, quantification cycle.

platelet-associated diseases (41). The formation and development of thrombi largely depends on platelet activation, in which a change in platelet parameters may affect the activation process (42). The MPV is the average measurement of platelet size, and as the platelet increases in size, the potential of thrombus formation increases (41).

The present study revealed that MPV values were increased by $10 \%$ in the pre-RT group in comparison with the control group and decreased by $6 \%$ in the pre-RT day 0 group and $10 \%$ in the pre-RT day 40 group comapred with the pre-RT group. These results indicated that MPV values were increased owing to cancer and decreased owing to RT. The MPV values were investigated in various types of cancer, including prostate cancer $(43,44)$; however, to the best of our knowledge, there are no studies investigating the change in MPV values associated with RT.

The platelet function markers investigated in the present study include platelet aggregation, a marker of platelet hyperactivity (45); P-selectin, an adhesion molecule, which is present in the alpha granules of platelets and released as a response to 

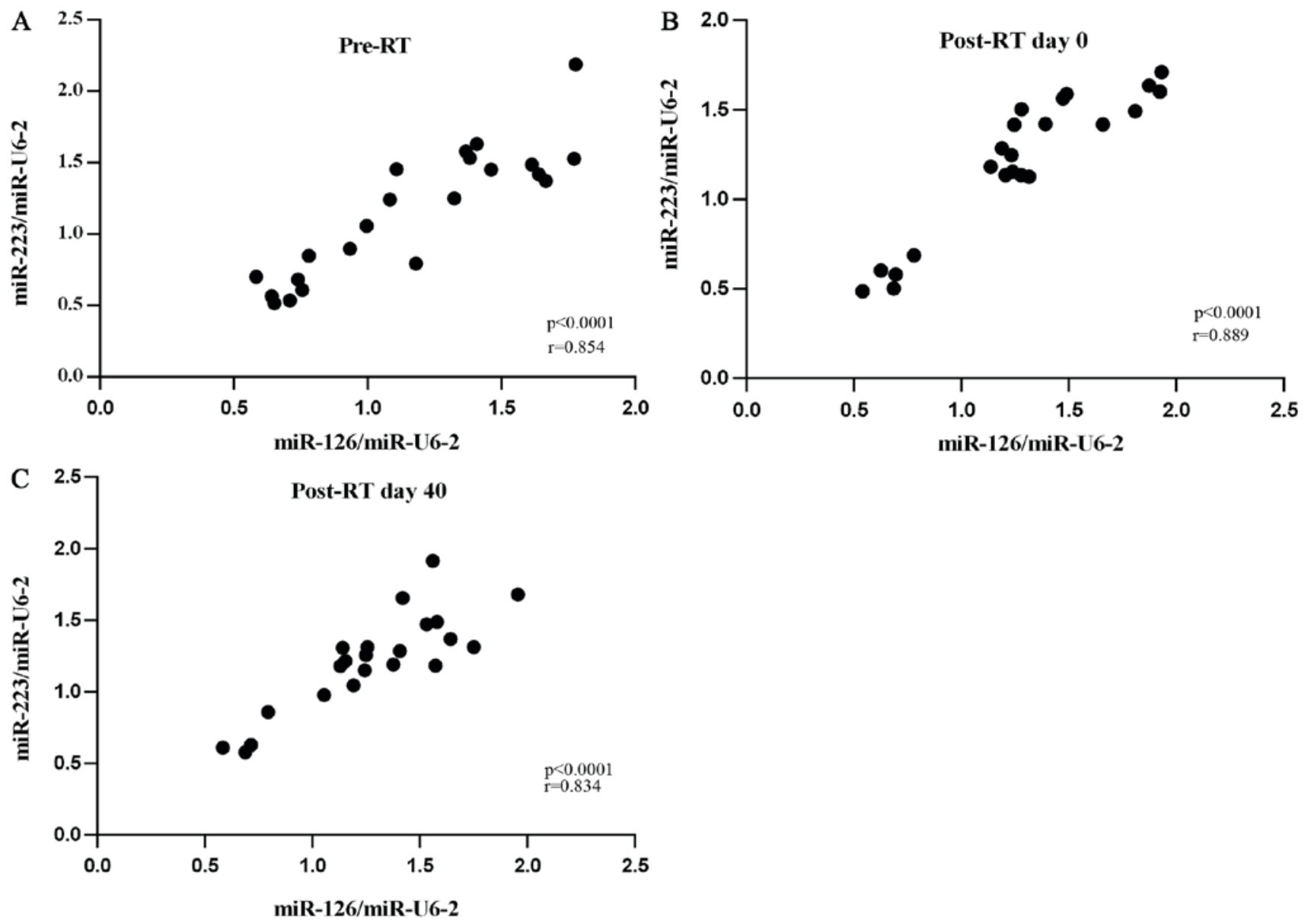

Figure 3. Correlation between Cq miR-223/Cq miR-U6-2 and Cq miR-126/Cq miR-U6-2 ratios in RT-treated groups. Correlation between Cq miR-223/Cq miR U6-2 and Cq miR-126/Cq miR U6-2 ratios in (A) the pre-RT group, (B) the post-RT day 0 group and (C) the post-RT day 40 group. RT, radiotherapy; miR, microRNA; Cq, quantification cycle.

cellular activities (46); thrombospondin-1, which is released during hemostatic plaque formation and an active player in thrombus formation (47), and PF 4, which serves a function in thrombosis and is released by platelet activation (48). When the data was evaluated, no significant change was identified in the levels of platelet aggregation, plasma P-selectin, thrombospondin-1 and PF 4 associated with RT between any of the RT groups.

Various studies reported a notable but unsignificant increase in platelet aggregation levels in vitro and in patients with metastatic prostate cancer compared with pre-RT conditions $(17,49)$. It has been suggested that extracorporeal irradiation does not affect the platelet functions that are already in systemic circulation, however, it may affect bone marrow platelet stem cells (17).

Experimental molecular studies that determine the effects of ionizing radiation on P-selectin levels revealed an increase in the concentration of P-selectin in association with RT $(18,33,50)$. However, Zekanowska et al (19) reported that the levels of soluble P-selectin do not change following RT in patients with prostate cancer. The results of the present study revealed that RT did not affect the plasma P-selectin concentration in patients with prostate cancer.

It has been revealed that the concentration of thrombospondin-1 changes owing to an increasing dose of radiation over time $(51,52)$. Lewinski et al (20) reported that thrombospondin-1 concentration did not change in association with the radioactive iodine treatment in patients with thyrotoxicosis.

Although contradictory results exist in clinical studies investigating PF 4 levels in various types of cancer (53-55), to the best of our knowledge no clinical studies have focused on the changes in plasma PF 4 levels associated with RT. A number of in vitro studies have provided evidence of the protective effect of PF 4 against the damaging effect of radiation $(56,57)$.

At present, the basis for the lack of effect of VMAT on platelet function remains unknown, although VMAT has a partial effect on the platelet count and MPV value. A potential explanation for this is that the highly developed dose distribution of VMAT may result in platelet activation in the target area below detectable levels in systemic circulation.

miRNAs are small molecules that target specific genes and regulate their transcription (57). In previous years, it has been revealed that miRNAs serve functions in various pathological and physiological processes (57).

A previous study has reported alterations in miRNA expression in different diseases, and that platelet miRNA levels were biologically and clinically important markers of the following: i) Platelet protein translation and expression; ii) mature megakaryocyte 
miRNA level; iii) hematological disease and platelet reactivity; and iv) basic mechanisms of megakaryocyte/platelets (58). Although the exact function of miRNAs in platelet function is not clearly understood, it is thought that miRNAs have notable functions in hemostasis and thrombosis (57).

Studies published in previous years provide evidence that a number of miRNAs are specific to cells and tissues and are differentially expressed $(24,59,60)$. It has been demonstrated that miRNAs can enter into systemic circulation via certain mechanisms, including exosome secretion, cell death and blebbing, and that these circulating miRNAs could be used as potential markers for and in the follow-up of certain diseases (24). So far, studies have focused on the changes of miRNA expression levels associated with cancer $(1,61)$. However, to the best of our knowledge there are no studies that have investigated the effects of radiation on platelet miRNA expression. In experimental and total body irradiation studies, it has been reported that the expression rates of a number of miRNAs, including miR-126 and miR-223, change in plasma and peripheral blood cells. miR-223 and miR-126 are miRNAs identified in platelets. It has been revealed that miR-223 targets the P2Y12 receptor that is present on platelets and inhibits it, thus suppressing platelet aggregation and activation (58). miR-126 is downregulated during megakaryocytopoiesis, indicating that it serves a function in differentiation (62).

Various studies have reported that changes in the plasma levels of miR-223 and miR-126 are associated with platelet function in cardiovascular diseases $(24,26,27)$. Considering the effect of radiation on platelet functions from these previous studies, a change in the expression levels of miRNAs may be expected to exert an effect on platelet activation-associated $\mathrm{RT}$. In vitro and experimental studies revealed that the expression levels of miR-223 and miR-126 changed over time with increasing radiation dose $(63,64)$. Following RT, changes to RT-associated serum/plasma level changes can be expected as platelets release miRNAs as RT damages the cells. These changes may depend on the type and duration of treatment and the dose distribution. Thus, the present study additionally investigated the expression levels of miR-223 and miR-126 in the plasma and the changes in the expression of these miRNAs following VMAT application.

The results of the present study did not reveal a significant change in miR-223 and miR-126 expression levels in association with VMAT in patients with prostate cancer. The lack of significant differences in miRNA expression between pre-RT and control groups may indicate that these miRNAs are not tumor-specific in serum/plasma. The VMAT technique achieved highly conformal treatment plans and decreased the normal tissue toxicity compared with other conventional RT techniques (65). Therefore, no significant changes in the miRNA expression levels were observed. Dynamic changes to miRNA expression levels in response to antitumor therapy may have resulted in this non-significant difference (66). However, in the present study, there existed a clear inter-individual variability to radiation response in terms of the expression of miRNAs. Notably, this situation may not be easily explained by previous observations. The cells of patients with cancer respond differently to RT, even when they are treated with the same curative dose. Furthermore, numerous different factors, including genetic variations, environmental stress, differences in nutritional state between patients and controls, and exposure to genotoxic chemical agents may affect individual responses to ionizing radiation (67-70). Therefore, further studies are required to understand the molecular events underlying the substantial inter-individual differences in miRNA expression in response to radiation.

There are a number of limitations to the present study, including its relatively small sample size, investigation of a limited number of miRNAs rather than all miRNAs associated with platelet activation and the focus on plasma samples. However, the levels of miRNA and activation markers in platelets may provide valuable data to evaluate the platelet function.

In summary, the present study demonstrated that there were no significant changes in platelet aggregation, plasma P-selectin, thrombospondin- 1 and PF 4 levels and miR-226 and miR-123 expression in plasma associated with RT. However, a significant change in platelet count and MPV values were identified to be associated with RT. The results of the present study indicated that VMAT may not be a risk factor for platelet activation in patients with prostate cancer, despite the fact that it reduced the platelet count and MPV values, which may also be a result of the suppressive effect of radiation on the hematopoietic system.

\section{Acknowledgements}

Not applicable.

\section{Funding}

The present study was supported by the Research Fund of the University of Istanbul (grant no. 376).

\section{Availability of data and materials}

The datasets used and/or analyzed during the present study are available from the corresponding author on reasonable request.

\section{Authors' contributions}

$\mathrm{NB}, \mathrm{IO}$ and BA were responsible for study conception and design. NB, İ, BA, OB, ST, FYA and MCA performed data analysis and interpretation. FYA provided the patient specimens. All authors approved the final version of this manuscript.

\section{Ethics approval and consent to participate}

The present study was approved by the Ethics Committee of the Cerrahpasa Medical Faculty of Istanbul University (Grant no. 28052/2012), and informed consent to participate in the study was obtained from all patients involved.

\section{Patient consent for publication}

No identifying patient information is included in the published manuscript. Participants provided their consent for the publication of the data. 


\section{Competing interests}

All the authors declare that they have no competing interests.

\section{References}

1. Sun X, Liu Z, Yang Z, Xiao L, Wang F, He Y, Su P, Wang J and Jing B: Association of microRNA-126 expression with clinicopathological features and the risk of biochemical recurrence in prostate cancer patients undergoing radical prostatectomy. Diagn Pathol 8: 208, 2013.

2. Ferlay J, Soerjomataram I, Dikshit R, Eser S, Mathers C, Rebelo M, Parkin DM, Forman D and Bray F: Cancer incidence and mortality worldwide: Sources, methods and major patterns in GLOBOCAN 2012. Int J Cancer 136: E359-E386, 2015.

3. He J, Hua J, Ding N, Xu S, Sun R, Zhou G, Xie X and Wang J: Modulation of microRNAs by ionizing radiation in human gastric cancer. Oncol Rep 32: 787-793, 2014.

4. Li M, Li GF, Hou XY, Gao H, Xu YG and Zhao T: A dosimetric comparison between conventional fractionated and hypofractionated image-guided radiation therapies for localized prostate cancer. Chin Med J (Engl) 129: 1447-1454, 2016.

5. Hayden AJ, Catton C and Pickles T: Radiation therapy in prostate cancer: A risk-adapted strategy. Curr Oncol 17 (Suppl 2): S18-S24, 2010

6. Mottet N, Bellmunt J, Bolla M, Briers E, Cumberbatch MG, De Santis M, Fossati N, Gross T, Henry AM, Joniau S, et al: EAU-ESTRO-SIOG guidelines on prostate cancer. Part 1 : Screening, diagnosis, and local treatment with curative intent. Eur Urol 71: 618-629, 2017.

7. Yang FE, Vaida F, Ignacio L, Houghton A, Nauityal J, Halpern H, Sutton H and Vijayakumar S: Analysis of weekly complete blood counts in patients receiving standard fractionated partial body radiation therapy. Int J Radiat Oncol Biol Phys 33: 617, 1995.

8. Verheij M, Dewit LG, Boomgaard MN, Brinkman HJ and van Mourik JA: Ionizing radiation enhances platelet adhesion to the extracellular matrix of human endothelial cells by an increase in the release of von Willebrand factor. Radiat Res 137: 202-207, 1994.

9. Hallahan DE, Chen AY, Teng M and Cmelak AJ: Drug-radiation interactions in tumor blood vessels. Oncology (Williston Park) 13 (10 Suppl 5): S71-S77, 1999.

10. Sørensen HT, Mellemkjaer L, Olsen JH and Baron JA: Prognosis of cancers associated with venous thromboembolism. N Engl J Med 343: 1846-1850, 2000

11. Kroll MH, Hellums JD, McIntire LV, Schafer AI and Moake JL: Platelets and shear stress. Blood 88: 1525-1541, 1996.

12. Ni H and Freedman J: Platelets in hemostasis and thrombosis: Role of integrins and their ligands. Transfus Apher Sci 28: 257-264, 2003.

13. Arcangeli S and Greco C: Hypofractionated radiotherapy for organ-confined prostate cancer: Is less more? Nat Rev Urol 13 400-408, 2016.

14. Zachariah B, Jacob SS, Gwede C, Cantor A, Patil J, Casey L and Zachariah AB: Effect of fractionated regional external beam radiotherapy on peripheral blood cell count. Int J Radiat Oncol Biol Phys 50: 465-472, 2001.

15. Lundgren MSFS, Cavalcanti MSM and Sampaio DA: Weekly monitoring of the effects of conventional external beam radiation therapy on patients with head and neck, chest, and pelvis cancer by means of blood cells count. Radiol Bras 41: 29-33, 2008

16. Ampil FL, Burton GV and Li BD: 'Routine' weekly blood counts during breast irradiation for early stage cancer: Are they really necessary? Breast J 7: 450-452, 2001.

17. Kalovidouris AE and Papayannis AG: Effect of ionizing radiation on platelet function in vitro. Acta Radiol Oncol 20: 333-336, 1981.

18. Mihaescu A, Thornberg C, Santén S, Mattsson S, Jeppsson B and Thorlacius H: Radiation-induced platelet-endothelial cell interactions are mediated by P-selectin and P-selectin glycoprotein ligand-1 in the colonic microcirculation. Surgery 151: 606-611, 2012.

19. Zekanowska E, Rystok-Grabska D, Wisniewski T, Ziolkowska E, Slomka A, Giemza-Kucharska P and Rosc D: C0141 Radiotherapy and platelets activation in patients with prostate cancer. Thromb Res 130: S163-S164, 2012.
20. Lewinski A, Brona A, Lewandowski KC, Jedrzejuk D, Bohdanowicz-Pawlak A, Skowronska-Jozwiak E, Bienkiewicz M and Milewicz A: Effects of radioiodine administration on serum concentrations of matrix metalloproteinases, adiponectin and thrombospondin-1. Thyroid Res 6: 9, 2013.

21. Lambert MP, Xiao L, Nguyen Y, Kowalska MA and Poncz M: The role of platelet factor 4 in radiation-induced thrombocytopenia. Int J Radiat Oncol Biol Phys 80: 1533-1540, 2011.

22. Willeit P, Zampetaki A, Dudek K, Kaudewitz D, King A, Kirkby NS, Crosby-Nwaobi R, Prokopi M, Drozdov I, Langley SR, et al: Circulating microRNAs as novel biomarkers for trombosit activation. Circ Res 112: 595-600, 2013.

23. Nagalla S, Shaw C, Kong X, Kondkar AA, Edelstein LC, Ma L, Chen J, McKnight GS, López JA, Yang L, et al: Platelet microRNA-mRNA coexpression profiles correlate with platelet reactivity. Blood 117: 5189-5197, 2011

24. de Boer HC, van Solingen C, Prins J, Duijs JM, Huisman MV, Rabelink TJ and van Zonneveld AJ: Aspirin treatment hampers the use of plasma microRNA-126 as a biomarker for the progression of vascular disease. Eur Heart J 34: 3451-3457, 2013.

25. Tian HS, Zhou QG and Shao F: Relationship between arterial atheromatous plaque morphology and platelet-associated miR-126 and miR-223 expressions. Asian Pac J Trop Med 8: 309-314, 2015.

26. Yu XY, Chen JY, Zheng ZW, Wu H, Li LW, Zhang ZW, Chen ZH, Lin QX, Han YL and Zhong SL: Plasma miR-126 as a potential marker predicting major adverse cardiac events in dual antiplatelet-treated patients after percutaneous coronary intervention. EuroIntervention 9: 546-554, 2013.

27. Zhang YY, Zhou X, Ji WJ, Shi R, Lu RY, Li JL, Yang GH, Luo T, Zhang JQ, Zhao JH, et al: Decreased circulating microRNA-223 level predicts high on-treatment platelet reactivity in patients with troponin-negative non-ST elevation acute coronary syndrome. J Thromb Thrombolysis 38: 65-72, 2014.

28. Angiolillo DJ, Fernandez-Ortiz A, Bernardo E, Ramírez C, Cavallari U, Trabetti E, Sabaté M, Jimenez-Quevedo P, Hernández R, Moreno R, et al: Lack of association between the $\mathrm{P} 2 \mathrm{Y} 12$ receptor gene polymorphism and platelet response to clopidogrel in patients with coronary artery disease. Thromb Res 116: 491-497, 2005.

29. Ruggeri ZM: New insights into the mechanisms of platelet adhesion and aggregation. Semin Hematol 31: 229-239, 1994.

30. Livak KJ and Schmittgen TD: Analysis of relative gene expression data using real-time quantitative PCR and the 2(-Delta DeltaC (T)) method. Methods 25: 402-408, 2001.

31. Burdak-Rothkamm S and Prise KM: New molecular targets in radiotherapy: DNA damage signalling and repair in targeted and non-targeted cells. Eur J Pharmacol 625: 151-155, 2009.

32. Mouthon MA, Vereycken-Holler V, Van der Meeren A and Gaugler MH: Irradiation increases the interactions of platelets with the endothelium in vivo: Analysis by intravital microscopy. Radiat Res 160: 593-599, 2003.

33. Hallahan DE, Staba-Hogan MJ, Virudachalam S and Kolchinsky A: X-ray-induced P-selectin localization to the lumen of tumor blood vessels. Cancer Res 58: 5216-5220, 1998.

34. Hallahan DE and Virudachalam S: Accumulation of P-selectin in the lumen of irradiated blood vessels. Radiat Re 152: 6-13, 1999.

35. Otto K: Volumetric modulated arc therapy: IMRT in a single gantry arc. Med Phys 35: 310-317, 2008.

36. Cozzi L, Dinshaw KA, Shrivastava SK, Mahantshetty U, Engineer R, Deshpande DD, Jamema SV, Vanetti E, Clivio A, Nicolini $G$ and Fogliata A: A treatment planning study comparing volumetric arc modulation with RapidArc and fixed field IMRT for cervix uteri radiotherapy. Radiother Oncol 89: 180-191, 2008

37. Nabavizadeh N, Simeonova AO, Waller JG, Romer JL, Monaco DL, Elliott DA, Tanyi JA, Fuss M, Thomas CR Jr and Holland JM: Volumetric-modulated arc radiotherapy for pancreatic malignancies: Dosimetric comparison with sliding-window intensity-modulated radiotherapy and 3-dimensional conformal radiotherapy. Med Dosim 39: 256-260, 2014.

38. Blank KR, Cascardi MA and Kao GD: The utility of serial complete blood count monitoring in patients receiving radiation therapy for localized prostate cancer. Int J Radiat Oncol Biol Phys 44: 317-321, 1999.

39. Pinkawa M, Djukic V, Klotz J, Petz D, Piroth MD, Holy R and Eble MJ: Hematologic changes during prostate cancer radiation therapy are dependent on the treatment volume. Future Oncol 10: 835-843, 2014. 
40. Kennedy AR, Maity A and Sanzari JK: A review of radiation-induced coagulopathy and new findings to support potential prevention strategies and treatments. Radiat Res 186: 121-140, 2016.

41. Mutlu H, Berk V, Karaca H, Erden A, Aslan T and Akca Z: Treatment regimen with bevacizumab decreases mean platelet volume in patients with metastatic colon cancer. Clin Appl Thromb Hemost 18: 546-548, 2012.

42. Khode V, Sindhur J, Kanbur K, Ruikar K and Nallulwar S: Mean platelet volume and other platelet volume indices in patients with stable coronary artery disease and acute myocardial infarction: A case control study. J Cardiovasc Dis Res 3: 272-275, 2012.

43. Riedl J, Kaider A, Reitter EM, Marosi C, Jäger U, Schwarzinger I, Zielinski C, Pabinger I and Ay C: Association of mean platelet volume with risk of venous thromboembolism and mortality in patients with cancer. Results from the vienna cancer and thrombosis study (CATS). Thromb Haemost 111: 670-678, 2014.

44. Inagaki N, Kibata K, Tamaki T, Shimizu T and Nomura S: Prognostic impact of the mean platelet volume/platelet count ratio in terms of survival in advanced non-small cell lung cancer Lung Cancer 83: 97-101, 2014.

45. Cooke NM, Egan K, McFadden S, Grogan L, Breathnach OS, O'Leary J, Hennessy BT and Kenny D: Increased platelet reactivity in patients with late-stage metastatic cancer. Cancer Med 2: 564-570, 2013.

46. Johnson RC, Mayadas TN, Frenette PS, Mebius RE, Subramaniam M, Lacasce A, Hynes RO and Wagner DD: Blood cell dynamics in P-selectin-deficient mice. Blood 86: 1106-1114, 1995.

47. Esemuede N, Lee T, Pierre-Paul D, Sumpio BE and Gahtan V: The role of thrombospondin-1 in human disease. J Surg Res 122 135-142, 2004

48. Sachais BS, Higazi AA, Cines DB, Poncz M and Kowalska MA: Interactions of platelet factor 4 with the vessel wall. Semin Thromb Hemost 30: 351-358, 2004.

49. Weiss K, Palumbo B, Palumbo I, Palumbo R, Granegger S, Hiltunen $J$ and Sinzinger $H$ : Platelet function after single [153Sm]EDTMP therapy in prostate cancer. Q J Nucl Med Mol Imaging 50: 330-333, 2006

50. El Kaffas A, Smith K, Pradhan P, Machtaler S, Wang H, von Eyben R, Willmann JK and Hristov D: Molecular contrast-enhanced ultrasound imaging of radiation-induced P-Selectin expression in healthy mice colon. Int J Radiat Oncol Biol Phys 97: 581-585, 2017.

51. Rofstad EK, Henriksen K, Galappathi K and Mathiesen B: Antiangiogenic treatment with thrombospondin-1 enhances primary tumor radiation response and prevents growth of dormant pulmonary micrometastases after curative radiation therapy in human melanoma xenografts. Cancer Res 63 : 4055-4061, 2003.

52. Rofstad EK, Galappathi K and Mathiesen B: Thrombospondin-1 treatment prevents growth of dormant lung micrometastases after surgical resection and curative radiation therapy of the primary tumor in human melanoma xenografts. Int J Radiat Oncol Biol Phys 58: 493-499, 2004

53. Wiesner T, Bugl S, Mayer F, Hartmann JT and Kopp HG: Differential changes in platelet VEGF, Tsp, CXCL12, and CXCL4 in patients with metastatic cancer. Clin Exp Metastasis 27: 141-149, 2010.

54. Poruk KE, Firpo MA, Huerter LM, Scaife CL, Emerson LL, Boucher KM, Jones KA and Mulvihill SJ: Serum platelet factor 4 is an independent predictor of survival and venous thromboembolism in patients with pancreatic adenocarcinoma. Cancer Epidemiol Biomarkers Prev 19: 2605-2610, 2010.
55. Lam YW, Mobley JA, Evans JE, Carmody JF and Ho SM: Mass profiling-directed isolation and identification of a stage-specific serologic protein biomarker of advanced prostate cancer. Proteomics 5: 2927-2938, 2005.

56. Chen JJ, Gao Y, Tian Q, Liang YM and Yang L: Platelet factor 4 protects bone marrow mesenchymal stem cells from acute radiation injury. Br J Radiol 87: 20140184, 2014.

57. Gatsiou A, Boeckel JN, Randriamboavonjy V and Stellos K MicroRNAs in platelet biogenesis and function: Implications in vascular homeostasis and inflammation. Curr Vasc Pharmacol 10: 524-531, 2012.

58. Edelstein LC, McKenzie SE, Shaw C, Holinstat MA, Kunapuli SP and Bray PF: MicroRNAs in platelet production and activation. J Thromb Haemost 11 (Suppl 1): S340-S350, 2013.

59. Corsten MF, Dennert R, Jochems S, Kuznetsova T, Devaux Y, Hofstra L, Wagner DR, Staessen JA, Heymans S and Schroen B: Circulating MicroRNA-208b and MicroRNA-499 reflect myocardial damage in cardiovascular disease. Circ Cardiovasc Genet 3: 499-506, 2010.

60. Wang GK, Zhu JQ, Zhang JT, Li Q, Li Y, He J, Qin YW and Jing Q: Circulating microRNA: A novel potential biomarker for early diagnosis of acute myocardial infarction in humans. Eur Heart J 31: 659-666, 2010.

61. Li X, Zhang Y, Zhang H, Liu X, Gong T, Li M, Sun L, Ji G, Shi Y, Han Z, et al: miRNA-223 promotes gastric cancer invasion and metastasis by targeting tumor suppressor EPB41L3. Mol Cancer Res 9: 824-833, 2011.

62. Garzon R, Pichiorri F, Palumbo T, Iuliano R, Cimmino A Aqeilan R, Volinia S, Bhatt D, Alder H, Marcucci G, et al: MicroRNA fingerprints during human megakaryocytopoiesis. Proc Natl Acad Sci USA 103: 5078-5083, 2006.

63. Templin T, Paul S, Amundson SA, Young EF, Barker CA, Wolden SL and Smilenov LB: Radiation-induced micro-RNA expression changes in peripheral blood cells of radiotherapy patients. Int J Radiat Oncol Biol Phys 80: 549-557, 2011.

64. Cui W, Ma J, Wang Y and Biswal S: Plasma miRNA as biomarkers for assessment of total-body radiation exposure dosimetry. PLoS One 6: e22988, 2011.

65. Jin L, Wang R, Jiang S, Yue J, Liu T, Dou X, Zhu K, Feng R, Xu X, Chen D and Yin Y: Dosimetric and clinical toxicity comparison of critical organ preservation with three-dimensional conformal radiotherapy, intensity-modulated radiotherapy, and RapidArc for the treatment of locally advanced cancer of the pancreatic head. Curr Oncol 23: e41-e48, 2016.

66. Ponomaryova AA,Morozkin ES, RykovaEY,ZaporozhchenkoIA, Skvortsova TE, Dobrodeev AY, Zavyalov AA, Tuzikov SA, Vlassov VV, Cherdyntseva NV, et al: Dynamic changes in circulating miRNA levels in response to antitumor therapy of lung cancer. Exp Lung Res 42: 95-102, 2016.

67. Smirnov DA, Brady L, Halasa K, Morley M, Solomon S and Cheung VG: Genetic variation in radiation-induced cell death. Genome Res 22: 332-339, 2012.

68. Foray N, Bourguignon M and Hamada N: Individual response to ionizing radiation. Mutat Res 770: 369-386, 2016.

69. Mei N, Imada H, Nomoto S, Kunugita $N$ and Norimura T: Individual variation and age dependency in the radiosensitivity of peripheral blood T-lymphocytes from normal donors. J Radiat Res 37: 235-245, 1996.

70. Barber JB, West CM, Kiltie AE, Roberts SA and Scott D: Detection of individual differences in radiation-induced apoptosis of peripheral blood lymphocytes in normal individuals, ataxia telangiectasia homozygotes and heterozygotes, and breast cancer patients after radiotherapy. Radiat Res 153: 570-578, 2000. 